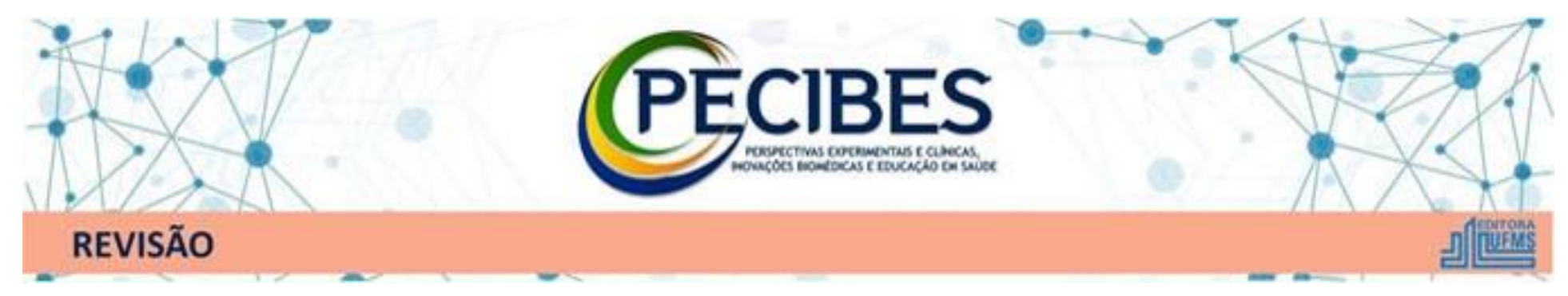

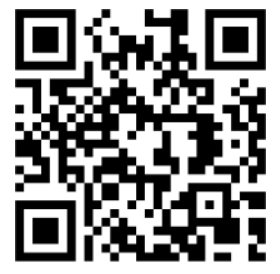

http://www.seer.ufms.br/index.php/p ecibes/index

*Autor correspondente: Luana Leão Chagas.

E-mail do autor:

luanachagas@msn.com

Palavras-chave: Bundle.

Pneumonia neonatal.

Key-words: Bundle. Pneumonia. Neonatal.

\section{Aplicação do bundle para pneumonia associada a ventilação mecânica em neonatologia: revisão integrativa}

Bundle application for pneumonia associated with mechanical ventilation in neonatology: integrative review

\section{Luana Leão Chagas ${ }^{1}$, Ana Maria Ortiz Azevedo², Sarah Cristina Silveira Santos ${ }^{3}$,}

Kely Cristina Garcia Vilena ${ }^{4}$, Karla de Toledo Candido Muller ${ }^{5}$

${ }^{1}$ Graduada do curso de Enfermagem da Universidade católica Dom Bosco

${ }^{2}$ Graduada do curso de Enfermagem da Universidade católica Dom Bosco

${ }^{3}$ Graduanda do curso de Enfermagem da Universidade católica Dom Bosco

${ }^{4}$ Prof. ${ }^{\circ}$ Ma. Docente do curso de Enfermagem da Universidade Católica Dom Bosco

${ }^{5}$ Prof. ${ }^{a}$ Dra. Docente curso de Fisioterapia da Universidade Católica Dom Bosco

\section{Resumo}

Objetivo: Avaliar estratégias utilizadas pela equipe multiprofissional nas Unidades de Terapia Intensiva Neonatal na prevenção de pneumonia associado à ventilação mecânica com o uso do Bundle. Materiais e métodos: Estudo de revisão integrativa de literatura. Para o levantamento dos artigos na literatura foi realizado uma busca nas seguintes bases de dados: SCIELO (Scientific Electronic Library Online), MedLine (Sistema Online de Busca e Analise de Literatura Médica) e LILACS (Literatura Latino-americana e do Caribe em ciência da saúde). Resultado e Discussão: A análise dos resultados nos permitiu verificar quatro principais estratégias na utilização do Bundle para prevenção da PAV, dentre elas: 1) Treinamento da equipe, 2) Boas práticas na utilização do Bundle, 3) Identificação de fatores de risco e medidas preventivas e 4) Implementação da extubação programada. Conclusão: Percebe-se que prevenir a PAV é uma intervenção diária e complexa, a qual necessita de profissionais capacitados e dispostos a estar em constante aprendizado sobre o tema em questão para melhor adequar as medidas preventivas de acordo com o serviço no qual estão inseridos.

\section{Abstract}

Objective: To evaluate strategies used by the multiprofessional team in Neonatal Intensive Care Units to prevent pneumonia associated with mechanical ventilation with the use of the Bundle. Materials and methods: Study of integrative literature review. For the survey of articles in the literature, a search was carried out in the following databases: SCIELO (Scientific Electronic Library Online), MedLine (Online System for Searching and Analyzing Medical Literature), and LILACS (Latin American and Caribbean Literature in Science) health). Result and Discussion: The analysis of the results allowed us to verify four main strategies in the use of the Bundle for the prevention of VAP, among them: 1) Training of the team, 2) Good practices in the use of the Bundle, 3) Identification of risk factors and measures preventive measures and 4) Implementation of programmed extubation. Conclusion: It is noticed that preventing VAP is a daily and complex intervention, which requires trained professionals who are willing to be constantly learning about the topic in question to better adapt the preventive measures according to the service in which they are inserted. 


\section{Introdução}

A Unidade de Terapia Intensiva Neonatal (UTIN) é um setor onde são admitidos pacientes de 0 a 28 dias de vida, que necessitam de cuidados intensivos, na maioria das vezes, recém-nascidos prematuros de baixo peso, com algumas complicações respiratórias, hepáticas ou cardíacos, portanto encontramos pacientes que necessitam de ventilação mecânica (VM), (Souza, 2017).

A VM é um procedimento onde o paciente necessita de assistência ventilatória prolongada ou controle da ventilação pulmonar. Por ser um procedimento invasivo, pode causar infecções relacionada à assistência à saúde (IRAS), pois a passagem do tubo oral à traqueia, pode levar bactérias, secreções e microrganismos aos pulmões, surgindo infiltração pulmonar novo ou progressivo, ocasionando pneumonia (Fior, 2017).

A Pneumonia associada à ventilação mecânica (PAV) trata-se de uma infecção pulmonar que acomete pacientes submetidos à intubação endotraqueal, após 48hs do procedimento. É a segunda causa mais comum de infecção relacionada à assistência da saúde, podendo prolongar o tempo de VM e aumentar a permanência hospitalar, além da mortalidade (Fior, 2017).

$\mathrm{Na}$ neonatologia o risco de PAV é maior, pois a colonização de microrganismo no recém-nascido se inicia no nascimento e tem relação direta com pelo menos dois mecanismos: (I) no parto normal os microrganismos presentes no canal vaginal migram para a cavidade bucal e, (II) no parto cesariano os microrganismos do ambiente e dos profissionais contaminam o recém-nascido (Pinto, 2019).

Diante disso é fundamental ter ações que possam prevenir essa incidência de PAV a fim de promover qualidade e segurança a assistência ao paciente sob ventilação mecânica dentro da UTIN. Para isso é necessário identificar os fatores de risco para PAV, classificados na literatura como modificáveis e não modificáveis. Os não modificáveis referem-se à idade, gravidade, doenças neurológicas, cirurgias, traumas, presença de comorbidade (insuficiência cardíaca, doenças pulmonares obstrutivas crônicas, diabetes, doenças neurológicas, neoplasias, traumas e prematuridade). Já os modificáveis são as intervenções que são relacionadas ao ambiente da UTIN, um ambiente propício a microrganismos, favorecendo a prescrição racional e dirigida dos antimicrobianos, sendo também vinculada a assistência à saúde, incluindo a vigilância microbiológica, Bundle e protocolos de prevenção (José, 2014).

Nesse contexto, para prevenção da PAV foi criado pelo CDC (Center For Disease Control And Prevention) um conjunto de medidas preventivas, o Bundle, caracterizado por um pacote que reúne intervenções e cuidados para melhoria da assistência à saúde na prevenção de PAV, promovendo um cuidado com qualidade e segurança ao paciente em ventilação mecânica na UTIN.

Partindo desse cenário, torna-se importante o estudo sobre a aplicação do bundle para analisarmos a importância das medidas preventivas, como o manuseio conforme a técnica asséptica, a higienização correta e o cuidado ao paciente. As mudanças de atitudes da equipe multiprofissional podem ser responsáveis na prevenção da pneumonia associado à ventilação mecânica. Nesse sentido, o objetivo do estudo foi avaliar na literatura as estratégias utilizadas pela equipe multiprofissional nas Unidades de Terapia Intensiva Neonatal na prevenção de pneumonia associado à ventilação mecânica com o uso do Bundle.

\section{Material e Métodos}

Este estudo trata-se de uma revisão integrativa da literatura, construída entre os meses de Janeiro a Agosto de 2020, a partir das bases de dados on-line: SCIELO (Scientific Electronic Library Online), MedLine (Sistema Online de Busca e Analise de Literatura Médica), LILACS (Literatura Latino-americana e do Caribe em ciência da saúde). Os critérios de inclusão foram: artigos primários, disponíveis no idioma português e inglês, artigos disponíveis na íntegra e sem custo. Foram excluídos artigos de revisão de literatura, teses, monografias e resumos.

Para seleção dos artigos foram utilizados os seguintes descritores: Bundle para pneumonia associado à ventilação mecânica neonatal, pneumonia associada à ventilação mecânica neonatal e unidade de terapia intensiva neonatal.

Para a realização dessa revisão integrativa utilizou-se as seguintes etapas, a saber: (I) busca nas bases de dados, (II) seleção dos artigos que atenderam aos critérios de inclusão disponíveis, (III) leitura dos artigos selecionados, (IV) análise e interpretação dos resultados, (V) discussão dos resultados.

\section{Resultados}

Foram encontrados 22 artigos, dos quais foram selecionadas 07 trabalhos que atenderam aos critérios de inclusão, do número total, 04 eram relacionados ao uso do Bundle para prevenção de PAV neonatal, 02 que associavam a Pneumonia com a VM neonatal e 01 que refere as complicações não clínicas da VM.

A análise dos dados nos permitiu verificar que dentre as pesquisas realizadas, três abordaram o uso do Bundle como ferramenta para prevenção da PAV (Perugini et al 2015; Cardoso e Bizani 2015; Azab et al 2015). Outras três ressaltaram as medidas de prevenção para a pneumonia sem aplicação do Bundle especificamente (José et al 2014; Wehb et al 2015; Barbosa et al 2006). E por fim, uma sétima pesquisa apresentou as boas práticas para prevenção da extubação não programada (ENP), uma das causas que tem como consequência a PAV (Pinto, 2019).

Os resultados obtidos dos artigos indicam que a taxa de pneumonia é reduzida significativamente após o treinamento entre a equipe, identificação dos principais fatores de risco, implementação do pacote de prevenção de PAV, pactuação do conceito de ENP entre a equipe, comunicação efetiva na equipe multiprofissional, fixação adequada do tubo no RN, aplicação adequada do bundle ( realizando vigilância de PAV, promover edução correta enfatizando a lavagem das mãos, manter cabeceira elevada entre 30-45 graus, monitorar sedação, realizar aspiração de secreção subglótica, realizar higiene oral, trocar circuito da VM e umidificadores, prevenir extubação acidental, monitorar pressão do cuff e antibioticoterapia).

\section{Discussão}

A análise dos resultados nos permitiu verificar quatro PECIBES, 2021, 01, 19-76| 19 
principais estratégias na aplicação do Bundle para prevenção especial da enfermagem, para mesclar competência técnica e da PAV, dentre elas: (1) Treinamento da equipe, (2) Boas humanização (Barbosa et al, 2006).

práticas na utilização do Bundle, (3) Identificação de fatores de risco e medidas preventivas e (4) Implementação da extubação programada.

\section{1. Treinamento em equipe}

Há uma importância muito significativa na qualidade do treinamento das equipes para a correta aplicação do bundle em diferentes formas de prevenção de comorbidades. Isso pode ser organizado na forma de palestra, simulações, reuniões e aulas para poder preparar de forma efetiva a equipe e o mais importante, trabalhar a sincronia dos profissionais a fim de que todos trabalhem com o mesmo objetivo, evitando a todo custo que comorbidades se instalem e possam elevar os riscos de mortalidade dos pacientes.

Como medida na aplicação do Bundle, o treinamento se torna relevante pois representa um importante passo nas buscas para melhorias na assistência e qualidade de vida ao paciente em questão. Tem como objetivo aprimorar as funçöes desenvolvidas pelos profissionais, valorizando e aperfeiçoando o papel de cada membro da equipe (Costa, 2015). A competência técnica, especificamente na equipe de enfermagem, está atrelada ao desenvolvimento e aprimoramento de habilidades cognitivas e manuais para uma assistência de enfermagem dirigida para a obtenção de resultados claros e visíveis (Cunha et al, 2009).

A educação continuada ou permanente no contexto da terapia intensiva é um fator determinante para que os resultados esperados sejam realmente alcançados e de maneira eficaz, segura e fidedigna fundamentadas na metodologia das práticas baseadas em evidências. Essa medida auxilia os profissionais a tomarem as decisões apropriadas no tratamento estabelecido dos pacientes críticos, desde que o programa e a organização desse instrumento sejam bem planejados, baseando-se em resultados observacionais e em processos padronizados e estratégias que reforçam o comportamento adequado de toda a equipe de assistência à saúde.

\section{2. Boas práticas na utilização do Bundle}

A equipe conhecendo os desafios a serem enfrentados, podem adotar estratégias para a diminuição das comorbidades associadas com a ventilação mecânica. Vale ressaltar a importância dessa equipe de enfermagem dentro de uma UTIN, já que ela estará assistindo o RN sob VM. A enfermagem deve estar ciente de que a monitorização respiratória à beira do leito é um item primordial, pois as mudanças ocorridas podem ser avaliadas através da alteração do padrão respiratório e da presença de ruídos adventícios na ausculta pulmonar.

A assistência humanizada ao paciente, requer um cuidado pautado na abordagem humanística, associando os saberes técnicos e científicos ao cuidado humano no qual está inserido o homem como um ser dotado de sentimentos e singularidades. Assim, o papel do enfermeiro é imprescindível, pois o cuidar é a sua essência. O neonato na UTIN requer cuidados especializados da equipe de saúde, em
Utilizando a ferramenta bundle, o enfermeiro se torna imprescindível junto aos demais profissionais nas orientações diárias para a manutenção de cabeceira do leito, da higiene oral, do posicionamento no leito, da pressão do cuff e dos demais cuidados essenciais para um bom prognóstico da ventilação mecânica.

De acordo com a construção do bundle, a higiene da cavidade oral com a clorexidine, deve acontecer com uma esponja e movimentos bem sutis, visto que a nutrição da pele já estará afetada e o risco de provocar lesões cutâneas é maior. A frequência normalmente deve ser de 3 a 4 vezes ao dia, onde os profissionais da equipe devem estar atentos às alergias, irritações na mucosa ou escurecimento transitório dos dentes (Beraldo e Andrade, 2008).

\section{3. Identificação de fatores de risco e medidas preventivas}

Os principais fatores de risco da associação entre VM e PAV são idade avançada (>70 anos); coma; nível de consciência, intubação e reintubação traqueal; extubação não programada, condições imunitárias; uso de drogas imunossupressoras; choque; gravidade da doença; doença pulmonar obstrutiva crônica (DPOC); tempo prolongado de VM; aspiração do condensado contaminado dos circuitos do ventilador; desnutrição; contaminação exógena; antibioticoterapia; colonização microbiana; cirurgias prolongadas; aspiração de secreções contaminadas; colonização gástrica e aspiração desta e o pH gástrico $>4$. Inclui-se na neonatologia prematuridade, baixo peso ao nascer, apgar baixo, nascidos pré-termo e hipotermia (Wehb et al, 2015).

Dentre as complicações não clínicas em VM no RN podemos citar: obstrução do tubo orotraqueal (TOT), sangramento traqueal por trauma durante as aspirações de secreções, lesões dérmicas, extubação acidental, escape ou queda de saturação de oxigênio, todas estas consideradas como fatores predisponentes a reintubações (Barbosa et al, 2006). A partir de tais fatores foram traçadas estratégias para diminuição dos índices de complicações. No entanto, a diminuição de complicações só é possível com o auxílio de toda equipe para a aplicação do bundle (Cardoso e Bizani 2015; Wehbe et al 2015).

Dentre as principais intervenções que o enfermeiro, junto aos demais membros da equipe podem atuar, têm-se a correção da cabeceira do leito, devido ao fato de a maioria dos pacientes internados em UTI neonatal possuírem o risco de broncoaspiração, principalmente quando estão expostos a manipulações das vias aéreas, como é o caso da ventilação mecânica. O paciente que está com via aérea artificial, estando impossibilitado de eliminar secreções de maneira natural com o mecanismo da tosse, haverá consequentemente um acúmulo de secreções nas vias aéreas que a longo prazo pode causar bradipnéia e consequentemente na troca gasosa servir de meio de cultura para microrganismos como bactérias e fungos.

Por isso, se houver migração dessa secreção contaminada para os pulmões, um quadro de pneumonia 
estará instalado, assim um dos principais objetivos do uso do bundle é impedir que isso aconteça. A maneira mais simples de evitar essa complicação é colocar a cabeceira do leito sempre em um ângulo inclinado entre $30^{\circ}$ e $45^{\circ}$ para prevenir a broncoaspiração e uma ventilação adequada ao paciente.

Estudos recentes mostraram uma diminuição significativa dos índices de broncoaspiração em pacientes que tinham a cabeceira elevada na angulação recomendada. Outros fatores contribuintes para evitar essa complicação é o correto posicionamento dos filtros e circuitos dos respiradores no nível da cabeceira do paciente, acima da comissura labial (Fior 2017; Perugini et al 2015; Cardoso e Bizani 2015; Azab et al 2015; Gonçalves et al 2015; Carvalheira et al 2020).

Outro fator participante do bundle é a pressão adequada do cuff, já que uma excessiva pressão pode comprometer a microcirculação da mucosa causando isquemia e uma baixa pressão pode dificultar a ventilação com pressão positiva, provocando um vazamento do ar na passagem da secreção subglótica entre o tubo e traqueia. A pressão deve estar em um nível ideal que seja suficiente para evitar futuras perdas de ar do circuito e a microaspiração do muco que fica acima do balonete. Segundo a literatura a pressão varia de paciente, mas deve estar sempre em uma escala entre 20 e $25 \mathrm{cmH} 2 \mathrm{O}$. Lembrando que os tubos orotraqueais para prematuros $<3 \mathrm{~kg}$ não são utlizados com cuff, os neonatos $>3 \mathrm{~kg}$ pode utilizar com cuff ou sem cuff, dependendo da estatura (Pinto 2019; Rodrigues et al 2020).

Como outros fatores agravantes, têm-se que pacientes intubados perdem o reflexo de defesa das vias aéreas e o refluxo esofágico, muito comum em pacientes com VM prolongada podendo levar à colonização endobrônquica e pneumonias em virtude da diminuição da ação bacteriana em meios de baixa concentração acidótica.

No que tange a higiene oral do paciente submetido a VM e sua importância é inquestionável para a prevenção de PAV, pois a falta de higienização oral ou de forma precária acaba por gerar a formação de placa bacteriana e consequentemente, colonização por microrganismo patógeno. De acordo com a literatura, o antisséptico mais utilizado na prevenção da PAV é o gluconato de clorexidine devido ao seu grande potencial bactericida, incluindo o combate a alguns germes resistentes (Oliveira, 2016).

A higiene oral de forma correta auxilia na diminuição da colonização bacteriana bucal, na prevenção e controle de infecções e manutenção da integridade da mucosa, além de proporcionar o devido conforto ao paciente.

\section{4. Implementação da extubação programada}

A relação entre o tempo de VM e a PAV são muito intrínsecas e para que isso não afete negativamente $o$ paciente, necessita-se um trabalho coeso dos profissionais de saúde, a fim de prever e antecipar a extubação de pacientes, evitando assim, as sedações desnecessárias, diminuindo os riscos de PAV. Normalmente, um paciente em VM necessita de uma sedação para o conforto e otimização do padrão ventilatório, mas uma sedação mais profunda e prolongada aumenta os riscos de infecções e retardam o desmame ventilatório. Além disso, observou-se também que a interrupção diária da sedação de forma gradativa está associada a uma maior sobrevida dos pacientes (Pinto, 2019).

Logo e de acordo com o bundle se faz necessário criar um protocolo de avaliação diária da sedação, avaliando também a prontidão neurológica para extubação e dentro desse protocolo construir uma alternativa que possa evitar a extubação acidental, tais como monitorizar e vigiar os sinais do paciente, podendo também implementar-se uma escala a fim de evitar aumento nos níveis de sedação.

\section{Agradecimentos}

Agradecemos a Universidade Católica Dom Bosco, em especial aos professores do Curso de Enfermagem por todo apoio e oportunidade de aprendizado.

\section{Declaração} trabalho.

Declaramos ausência de conflito de interesse neste

\section{Referências}

Azab SFA, Sherbiny HS, Saleh SH, Elsaeed WF, Elshafiey MM, Siam AG, Arafa MA, Alghobashy AA, Bendary EA, Basset MAA. Reducing ventilator-associated pneumonia in neonatal intensive care unit using "VAP prevention Bundle": a cohort study. Bmc Infectious Diseases, Zagazigue, 06 ago. 2015; 15, (1), 1-7.

Barbosa AL, Campos ACS, Chaves EMC. Complicações não clínicas da ventilação mecânica: ênfase no cuidadoi de enfermagem neonatal. Acta Paul Enferm 2006; 19, (4), 439-43.

Beraldo CC, Andrade D. Higiene bucal com clorexidina na prevenção de pneumonia associada à ventilação mecânica. Jornal Brasileiro de Pneumologia, Ribeirão Preto, 2008; 34, (9): 707-714.

Brasil. Agência Nacional de Vigilância Sanitária, Medidas de Prevenção de Infecção Relacionada à Assistência à Saúde. Brasília: Anvisa, 2017.

Cardoso MEV, Bizani D. Aplicação de bundle de prevenção de pneumonia associada à ventilação mecânica em centro de terapia intensiva adulto: um relato de experiência. Saúde e Desenvolvimento Humano, Canoas, nov. 2015; 3, (2): 137, 27.

Cardoso VB. | Entendimento dos enfermeiros intensivistas sobre as formas de prevenção de pneumonia associada à ventilação mecânica invasiva: Revisão da literatura. Rev. Eletrôn. Atualiza Saúde | Salvador, jan./jun. 2015; 1, (1).

Carmo N, Edgard S, Paulo C, Azevedo F, Lugarinho ME. Pneumonia associada à ventilação mecânica: análise de fatores epidemiológicos na confecção de estratégias de profilaxia e terapêutica. Revista Brasileira de Terapia Intensiva, São Paulo, dez. 2006; 18, (4): 344-350.

Carvalheira PLS, Buffel TS, Souza DA, Almeida ICJ, Barbosa MTS R, Messias C M. O Enfermeiro na prevenção da 
pneumonia associada à ventilação mecânica em unidade de terapia intensiva neonatal. Saúde Coletiva, Barueri, 2020; 9, (50): $1803-1809$.

Costa JB, Lima ALC, Torres F, Silva AFG, Terra J, André T. Os principais fatores de risco da pneumonia associada à ventilação mecânica em uti adulta. Revista Científica Faema, 19 jul 2016; 7, (1): 80-92.

Cunha, Lissandra Borba; Valério, Selma Tavares. Treinamento. 2009. 2, Instituto Israelita De Ensino E Pesquisa Albert Einstein, São Paulo, 2009.

Fior R. Prevention of ventilator-associated pneumonia: Nursing Course Conclusion Paper - Anhanguera, Campo Grande, pages. 27. 2017.

Gonçalves RL, Tsuzuki LM, Carvalho MGS. Endotracheal suctioning in intubated newborns: an integrative literature review. : an integrative literature review. Revista Brasileira de Terapia Intensiva, São Paulo, ago. 2015; 27, (3): 284-292.

Costa DB da, Garcia SD, Vannuchi MTO, Haddad MCL. Impacto Do Treinamento De Equipe No Processo De Trabalho Em Saúde: Revisão Integrativa, Revista De Enfermagem Ufpe On Line, Recife: Reuol, Abr. 2015.

José JD, Lucio DS, Perugini MR, Stipp AT, Silva, Louise MF, Perugini VH, Capobiango JD. Prevenção de pneumonia associada à ventilação mecânica em neonatologia. Revista Journal Of Infection Control, Londrina, nov. 2014; 4, (1): 20-24.

Miyaki M. Monitoramento microbiológico seqüencial da secreção traqueal em pacientes intubados internados em unidade de terapia intensiva pediátrica. J. Pediatr. Rio Janeiro, fevereiro. 2005; 8, (1): 3-4.

Oliveira TC. Eficácia da higiene oral na prevenção de pneumonia associada à ventilação mecânica. Biológicas \& Saúde, Campos dos Goytacazes, 31 ago. 2016; 21, (6): 20-24.

Perugini MRE, Perugini VH, Figueira FD, Fontana LMS, Diniz JJ, Santos DL, Belei RA, Vespero EC, Pelisson M, Stippabe AT. Impacto de um bundle nas taxas de pneumonia associada à ventilação mecânica (PAV) em uma unidade de terapia intensiva pediátrica em Londrina-PR. Semina: Ciências Biológicas e da Saúde, Londrina, ago. 2015; 36, (1): 259-266.

Pinto MM. Intervenções de enfermagem na prevenção de extubação não programada em recém-nascidos: bundle de boas práticas. Revista Enfermagem em Foco, Fortaleza, 06 set. 2019; 10, (7): 115-120.

Rodrigues JPBB, Bacci SLLS, Pereira JM, Johnston C, Azevedo VMGO. Frequência e caracterização da utilização de cânulas traqueais com balonete em unidades de terapia intensiva neonatais e pediátricas do Brasil. Rev. bras. ter. intensiva, São Paulo, Apr./June 2020 Epub. /July 13, 2020; 32, (2).

Silva DG. A importância da higienização das mãos na redução de infecções em serviços de saúde. 2012. 14 f.
Monografia (Especialização) - Curso de Farmácia, Puc/goiás, Goiás.

Silva VFF. Uso do Método PDSA para a elaboração e implementação de um protocolo de prevenção de pneumonia associada à ventilação mecânica em uma Unidade de Terapia Intensiva [dissertation]. Ribeirão Preto: University of São Paulo, Escola de Enfermagem de Ribeirão Preto; 2018.

Sousa DS, Sousa JAS, Santos ADR, Melo EV, Lima SO, Almeida SMA. Morbidade em recém-nascidos prematuros de extremo baixo peso em unidade de terapia intensiva neonatal. Rev. Bras. Saude Mater. Infant. [Internet]. Mar. 2017; 17, (1): 139-147.

Wehbe MAM, Lustosa SAS, Rocha APF, Oliveira, IVD. Pneumonia associada à ventilação mecânica em neonatologia: um estudo retrospectivo. Residência pediátrica, Volta Redonda. 2015; 5, (3): 118-121. 